\title{
SOFT POWER COMO ESTRATÉGIA DE MARKETING: A MANIFESTAÇÃO DA CULTURA POP JAPONESA NO BRASIL
}

\author{
Jaqueline Naomy Isshiki ${ }^{1}$ \\ Silvio Yoshiro Mizuguchi Miyazaki
}

Resumo: O objetivo do artigo é verificar a utilização de Soft power como estratégia de marketing, e a manifestação da cultura pop japonesa no Brasil. Para tanto, o artigo conceitua Soft Power e sua relação com a estratégia de marketing de um país, conhecida como Nation Branding. Posteriormente, analisa-se a dimensão cultural do Nation Branding do Japão, e o desenvolvimento do projeto pelo governo japonês para a imagem da marca do país: o Cool Japan.

Palavras-chave: Soft power, Marketing, Governo Japonês, Cool Japan, Nation Branding

Abstract: This paper verifies the use of soft power as a marketing strategy, and the manifestation of the Japanese pop culture in Brazil. Therefore, this paper conceptualizes Soft Power and its relation with the country marketing strategy, known as Nation Branding. Subsequently, the paper analyzes the cultural dimension of Japan's Nation Branding, and the development of the project called Cool Japan.

Keywords: Soft power, Marketing, Japanese Government, Cool Japan, Nation Branding

1. Bacharel em Marketing pela Escola de Artes, Ciências e Humanidades da Universidade de São Paulo (EACH-USP), São Paulo, Brasil; jaqueshiki@hotmail.com

2. Professor Doutor de Economia na Escola de Artes, Ciências e Humanidades (EACH-USP) e no Programa de Pós-Graduação em Língua, Literatura e Cultura Japonesa da Universidade de São Paulo (USP), São Paulo, Brasil; symiyazaki@usp.br 


\section{Introdução}

O Japão, após o final da Segunda Guerra Mundial, teve altas taxas de crescimento econômico, de 1946 a 1955 fora de 10,56 \% ao ano, na década seguinte, de 1955 a 1965, de $9,51 \%$ ao ano. De 1965 a 1975 de 7,85 \% ao ano (FLATH: 89, 2005). Esse crescimento propiciou o desenvolvimento do país de tal forma que se acreditava que o Japão superaria os Estados Unidos (VOGEL, 1979). De 1968 até 2010, o Japão fora a segunda maior economia do mundo, atrás somente dos Estados Unidos, sendo superada pela China nesse ano.

A partir dos anos de 1980, ficou forte a imagem associada aos produtos japoneses de que são de qualidade e tecnologicamente avançados; mesmo o modelo japonês de administração foi muito estudado, assim como as técnicas de produção utilizadas nas fábricas.

Entretanto, a partir de meados da década de 1990, a economia japonesa perdeu o seu vigor. Nos últimos anos, o Japão tem enfrentado crescimento de níveis modestos à estagnação econômica, de 1995 a 2000 a taxa média de crescimento fora de $0,83 \%$, e isso se refletiu também em suas relações com outros países. Nessa conjuntura, o Japão tenta recuperar sua influência e importância de outras formas, através de recursos denominados como soft power, em que Nye (1990) define como o poder que um país possui para influenciar indiretamente o comportamento de outros países através de recursos que não a força militar ou econômica, por exemplo, através de atrações culturais, ideologia e instituições internacionais. Segundo o ex-ministro do Japão, Taro Aso, o seu país está buscando melhorar a imagem regional e global, através da cultura pop como instrumento diplomático. Entre eles podemos citar: mangá, animê, música e a moda.

Em 2011, após as tragédias que assolaram o país (terremoto, tsunami e crise nuclear) foi lançada a proposta "Cool Japan Strategy" pelo Ministério da Economia, Comércio e Indústria e o "Cool Tokyo", com o propósito de expandir a disseminação e influência da cultura japonesa pelo mundo e revitalizar a imagem do país através da moda, culinária, conteúdo, produtos regionais, habitação e turismo. Ambas foram estratégias voltadas para solucionar problemas em momentos de crise através da criatividade, baseadas nas ideias do governo de Blair, na Inglaterra em 1997. Para Blair, uma medida de longo prazo para reconstruir a imagem do país seria torná-lo mais ligado à cultura pop e jovem (YOSHIMOTO, 2003).

Este artigo tem como objetivo verificar a utilização no Brasil pelo governo japonês de soft power, como uma estratégia de marketing. Para isso, o artigo está organizado em quatro seções: na seção 1, conceitua-se Soft Power; em seguida, a seção 2 analisa a sua relação com a estratégia de marketing de país conhecida como Nation Branding. A seção 3 aborda a principal dimensão de Nation Branding do Japão, a cultural, na qual foi desenvolvido um projeto pelo governo japonês, a fim de trabalhar a imagem da marca Japão: o Cool Japan. A seção 4 trata da experiência da cultura pop japonesa no Brasil. Por fim, analisa-se a atual efetividade deste projeto. 


\section{Soft Power}

O conceito de Soft Power, ou poder brando, foi criado por Nye nos anos de 1990. O soft power emana de três recursos: a cultura, os valores políticos e política externa. (NYE, 1990). O conceito surgiu no cenário pós Guerra Fria; o seu intuito era servir como um novo modelo de atuação para a busca de poder internacional, através da imagem e da cultura. Nye define soft power como a habilidade dos outros fazerem o que você quer através da atração. É diferente do hard power, que consiste na capacidade que os outros façam o que você quer por meio da coerção, muitas vezes relacionadas à força militar e recursos econômicos. Um exemplo de hard power seria uma ação militar, e de soft power seria uma exportação cultural do país, tais como os desenhos animados japoneses (SNOW, 2008).

O fenômeno do soft power foi criado no período conhecido como a fase de "grande transformação", período que representa um momento de transição entre a Guerra Fria, palco da disputa ideológica entre Estados Unidos e União Soviética, e a formação da nova ordem internacional (ALMEIDA, 2008).

Em discurso proferido no dia 30 de junho de 2009 pelo ex-primeiro ministro do Japão, Taro Aso fala das variadas formas de soft power praticadas pelo país:

O Japão ostenta uma gama ampla e variada de soft power que inclui a ética do trabalho japonês, graças à qual este país foi capaz de se recuperar das ruínas da derrota na guerra para se tornar uma superpotência econômica, como o estilo de trabalho japonês de sempre cumprir prazos de entrega e técnicas de excelência na fabricação de produtos de valor.

[...] O idioma japonês também é uma forma de soft power. Números cada vez maiores de pessoas em todo o mundo passaram a interessar-se pelo aprendizado do idioma japonês, dentro de um contexto de interesse pelo Cool Japan. Há até mesmo jovens que começaram a estudar japonês para serem capazes de ler as orientações dos jogos de computadores, que mostram formas para aumentar suas chances de vencer (ASO, 2009).

\section{Soft Power e Marketing: Nation Branding}

Relacionado ao soft power há uma ferramenta denominada nation branding, ou branding de países, que pode ser considerada importante no desenvolvimento do soft power uma vez que promoverá uma imagem mais favorável na comunidade internacional, preocupando-se com a imagem de um país de forma mais ampla, ou seja, nas dimensões política, econômica e cultural. (FAN, 2010).

De forma resumida, podemos definir nation branding como políticas de promoção que compara um país a uma marca. O Japão, por exemplo, pratica a nation branding através do projeto denominado Cool Japan. Podemos definir marca como "um conjunto multidimensional de elementos funcionais, emocionais, relacionais, e estratégicos que coletivamente geram um conjunto único de associações na mente do público"(AAKER, 1996, p.68). Fan (2006, p.2) coloca que "o branding de países pode ser definido 
como a soma total de todas as percepções de um país na mente dos stakeholders internacionais, que podem conter alguns dos seguintes elementos: pessoas, locais, cultura/língua, história, alimentação, moda, caras conhecidas, marcas globais, etc”. Utiliza-se de técnicas de comunicação, de criação de marca e mercado com o objetivo de promover a imagem de um país (FAN, 2006). Dinnie (2008) afirma que o nation branding dispõe de elementos que propiciam a um país a capacidade de diferenciar-se de outros atores, ou seja, permite a ele uma vantagem competitiva que incentiva visitar o país em questão. Assim como qualquer marca comercial, o nation branding tem seus "consumidores" que, no caso, são representadas pelas populações estrangeiras; o seu objetivo é exatamente estabelecer uma comunicação para esse público, podendo ser direcionado para: atrair investimento estrangeiro, atrair turistas, reposicionar a imagem do país tornando-a positiva, entre muitos outros. O nation branding é composto por seis dimensões: turismo, exportação, governo, investimento e imigração, cultura e história e pessoas (ANHOLT, 2009). No caso do Japão, a cultural é a principal dimensão dos esforços de nation branding.

\section{Cool Japan}

Criada com o intuito de expandir a influência do país nos campos cultural, econômico, social e político para outros países, uma estratégia de política internacional proposta pelo governo japonês foi o Cool Japan. O termo teve origem no artigo Japan's Gross National Cool, publicada na revista Foreign Policy por Douglas McGray em 2001. No artigo, McGray cita o potencial do Japão em se tornar uma superpotência cultural, deixando um pouco de lado aquela imagem mais conservadora, dando lugar a um país com música pop, eletrônicos, arquitetura, moda, animação e gastronomia. No artigo, ele mostra-se surpreendido pela rápida recuperação do Japão pós-crises econômicas e políticas. Apontou como os principais elementos nipônicos que ficaram conhecidos internacionalmente, o sumô, a gastronomia e a moda. Para ele, toda essa cultura "cool" foi impulsionada pela recessão (MCGRAY, 2001).

O Cool Japan foi uma medida tomada pelo governo para melhorar a imagem do país, que tem sido abalada por eventos históricos, tais como participações em guerras, atuação de governos anteriores, problemas com outros países asiáticos. Esses eventos fizeram o Japão ter uma imagem de país conservador e pouco amigável. Com o Cool Japan, o Japão reconstruiria sua imagem, gerando condições favoráveis na sua relação com o mundo. Sendo assim, a circulação de produtos japoneses no mundo, inspiraria consumidores do globo ansiando por produtos de origem japonesa, com isso o país ampliaria as exportações de seus produtos culturais (ALLISON, 2009).

Taro Aso, em discurso proclamado em abril de 2009, fala sobre o projeto Cool Japan. Ele fala que o país é uma grande fonte de conteúdo de entretenimento e moda, e isso fez com que o Japão ganhasse a atenção do mundo todo, seja com suas animações, 
jogos de computador, mangás ${ }^{3}$, moda e o mundo eletrônico de Harajuku e Akihabara ${ }^{4}$, Apesar disso, ele conta que esse soft power dessa indústria de conteúdo não gerou grandes negócios no exterior, representando menos de $2 \%$ de todas as vendas externas do Japão. Os planos do ex-ministro do Japão eram em investir nessa indústria, com a criação de um órgão que compraria as licenças para scripts de criadores populares, desenvolver canais de vendas e fornecer capital para a criação de obras no exterior de forma integrada, proporcionando incentivos à expansão dessa indústria de audiovisual e produção da cultura pop. Através da internet e dos mobile phones, desenvolver-se-ia obras e talentos transformando tais conteúdos em negócios ${ }^{5}$.

Além disso, o ex-ministro, a fim de pulverizar a cultura pop japonesa, propôs criar um prêmio para os artistas de mangá não-japoneses e introduzir no exterior os "Embaixadores culturais do Animê", representado atualmente pelo personagem Doraemon (MONJ, 2010). Além desses projetos, outro projeto implementado pelo MOFA, o Ministério de Negócios Estrangeiros do Japão, foi a nomeação de três "embaixadoras" para o título de "Comunicadores de moda da Cultura Pop Japonesa", ou mais conhecidas como embaixadoras do kawaii, termo designado para referir-se a coisas fofas ou meigas, desde o estilo Lolita até os mais ousados estilos que são possíveis encontrar em Harajuku. (HIGHLIGHTING JAPAN, 2009)

Para a realização do projeto Cool Japan, o governo criou um fundo de US\$500 milhões (HONG, 2014; JAPAN, METI, 2014). Esse projeto, basicamente, tem o intuito de melhorar a imagem do país e torná-lo atrativo, e com isso exportar sua cultura pop. Segundo o MOFA e a Fundação Japão, a cultura pop japonesa é caracterizada por: animê media (mangá, animê, videogames), música, TV e filmes, e moda japonesa, descritos a seguir.

\section{a. Animê media}

Pode-se dizer que uma das grandes conexões do Japão com o mundo são os animês e mangás. Em um período de dez anos — entre 1993 e 2003 — as exportações culturais japonesas triplicaram, chegando a 12,5 bilhões de dólares, sendo a indústria da animação (quadrinhos, desenhos animados e videogames) o maior impulsionador dessas exportações (GRAIEB, 2004).

$\mathrm{O}$ animê media acumula por ano de receita o equivalente a US\$ 2,1 bilhões, incluídas da TV, cinema, DVD e internet. Além disso, o varejo especializado em vender produtos baseados nos personagens de mangá e animê faturou em 2006 o equivalente a US\$13 bilhões (ONOUCHI, 2007). Mangá são as histórias em quadrinhos no estilo japonês, e um dos principais meios de divulgação da cultura e língua japonesa ao redor do mundo. O termo animé é uma abreviação da palavra japonesa (animê - shon), é derivada da palavra em inglês animation, que corresponde aos desenhos animados (SATO, 2007). Osamu Tenzuka é um grande nome para este universo. Ele foi o responsável, através

\footnotetext{
3. Histórias de quadrinhos japoneses.

4. Bairros em Tóquio.

5. Discurso disponibilizado em http://japan.kantei.go.jp/asospeech/2009/04/09speech_e.html
} 
de seus mangás por convencionar características dos animês que vemos atualmente, ou seja, com o uso de uma linguagem e estética cinematográfica conjugada à serialização das histórias (GRAVETT, 2006). Nessa indústria, vale destacar também um grande nome da animação cinematográfica que contribuiu muito para a imagem do país: Hayao Miyazaki com filmes como Meu vizinho Totoro, 1989; O Castelo Animado, 2004; e o ganhador do Oscar de 2003 como o melhor longa-metragem animado, A viagem de Chihiro, 2001.

\section{b. Música}

A respeito da música pop japonesa vale mencionar o J-Pop, abreviação de Japanese Popular Music, que é música japonesa influenciada por ritmos populares ocidentais (SATO, 2007). Ayumi Hamasaki, BoA, Utada Hikaru e Amuro Namie são algumas das principais cantoras desse estilo, e dentre as boy-bands podemos citar o grupo KAT-TUN. No gênero J-Rock vale citar as bandas L'arc en Ciel e Orange Range.

$O J$-music (que inclui todos os gêneros da música japonesa, como o pop e o rock) movimenta uma indústria fonográfica, considerada a segunda maior do mundo, atrás somente dos Estados Unidos (ALBUQUERQUE, 2015).

Relacionado ao Animê Media, existe os Animê Songs, que inclui os mais variados gêneros da música japonesa, mas que se caracterizam por serem canções-tema de séries, filmes e animações, podendo ser interpretadas por dubladores e cantores especializados nesse ramo, assim como por figuras populares da J-Music (NAGADO, 2007).

Existem cantores que são verdadeiros personagens de animê e estão fazendo bastante sucesso na web. Hatsune Miku é a mais famosa delas, que corresponde, na verdade, a uma versão de Vocaloid. O Vocaloid é um programa que possibilita a sintetização de voz com tecnologia. O programa funciona como uma biblioteca de sons e propicia liberdade ao usuário em compor músicas ou fazer novas versões de vozes de personagem já existentes e criar videoclipes (KENMOCHI, 2010).

\section{c. Moda}

Foi a partir da década de 50 que houve uma reviravolta da moda tradicional japonesa para a atual moda urbana, que é visivelmente perceptível nas ruas dos centros de Harajuku e Shibuya ${ }^{6}$. A moda urbana japonesa é uma mistura de elementos socioculturais ocidentais e orientais. As roupas são muito marcantes e distinguem os vários microgrupos presentes no país. Dentre elas, podemos citar o estilo Kogaru, caracterizado por um visual semelhante ao de uma colegial rebelde, usando minissaia e cabelos descoloridos; o Ganguro que se caracteriza pelo tom de pele altamente bronzeado de forma artificial, que contrasta com a maquiagem branca em certos pontos da face, e o uso de botas plataformas; o Gyaru que tem Paris Hilton como inspiração de looks, com muita maquiagem e o uso de lentes que aumentam a retina para se parecer com os olhos ocidentais; o Lolita que se assemelham às bonecas francesas de estilo

6. Bairros em Tóquio. 
vitoriano; o Visual Kei que é marcado por um visual mais gótico e sombrio, sem deixar de ser extravagante; é o estilo característico de bandas de $J$-rock (rock japonês); o Decora onde os adeptos desse estilo vestem tudo o que há de colorido, sem limites; e o Mori Girl, também chamado de Dolly Kei que reinventa looks com roupas de segunda mão (MACIAS e EVERS; 2007).

\section{A experiência da cultura pop japonesa no Brasil}

O primeiro contato da produção cultural japonesa em mídia televisiva brasileira foi a transmissão da série National Kid em 1964, pela emissora Record; o sucesso trouxe aos brasileiros a imagem do super-herói japonês (SATO, 2007). Nos anos 80, as séries japonesas repletas de super-heróis, monstros gigantes e robôs ganharam o gosto dos brasileiros e fizeram muito sucesso (PEREIRA, 2008), como Jaspion e Changeman. Essas séries, chamadas de live-action, abriram as portas para os animês nas TVs brasileiras (VILIEGAS, 2001).

Os animês chegaram ao Brasil por volta da década de 1960 na TV aberta com o animê Oitavo Homem, mas foi só a partir de 1994, com um investimento bilionário do governo japonês, que começou a conquistar fãs brasileiros. O primeiro grande sucesso a eclodir no Brasil foi a animação Os Cavaleiros do Zodíaco, transmitido pela TV Manchete no segundo semestre de 1994. O sucesso impulsionou a venda de brinquedos e revistas especializadas sobre o desenho. Além desse, outros animês que geraram grande repercussão em terras brasileiras foram: Dragon Ball e Pokémon. O Brasil é um dos países que mais recebeu títulos de animês (NAGADO, 2007). Apesar disso, dos anos 60 até hoje, os animês transmitidos pela TV brasileira, tanto aberta quanto fechada, têm desagrado os fãs, que anseiam por variedade e qualidade, uma vez que muitas cenas são censuradas pelas emissoras, e esse tipo de mediação pelas distribuidoras interfere na experiência dos brasileiros que consomem esses produtos.

Por sua vez, os mangás só chegaram ao Brasil no final dos anos 80 para os 90 com Lobo Solitário, em 1988, seguido de Akira dois anos depois. De acordo com Oka (2005), houve uma pausa de dez anos nas publicações de mangás, devido à falta de repercussão. Somente a partir de 1999, com a chegada de animês como Pokemon, Samurai X, Dragon Ball Z e Sakura Card Captors a situação mudou. Os animês e live actions impulsionaram as vendas de mangás, atingindo um número de 30 exibições desses gêneros nos canais brasileiros. No início, os mangás eram distribuídos em formato ocidental, como os livros que conhecemos, ou seja, com a leitura da esquerda para a direita. A partir de 2000, as editoras resolveram lançá-los com a manutenção do formato oriental, com a leitura da direita para esquerda. Com os títulos importados, portanto, com a necessidade de pagamento de direitos autorais entre outras despesas, com a desvalorização da moeda brasileira em relação ao dólar em 2002, o mercado editorial sofreu queda nas vendas de histórias de quadrinhos, mas o fenômeno não afetou a venda de mangás, que teve um aumento no número de títulos (GOTO, 2003). 
As principais editoras que trouxeram os mangás para o público brasileiros são: Conrad, JBC, Panini e NewPop, que somaram juntas 105 títulos de mangás à venda nos sites das editoras no ano de 2007. Para Gusman (2005), o mangá chegou ao país e assumiu um papel de atender à demanda de novos leitores, que não estavam acostumados a consumir as histórias de quadrinhos americanas, e representava a principal fatia do mercado nacional de quadrinhos. Em 2007, os exemplares que circulavam pelo país de mangás pela Conrad e JBC somavam 140 mil por mês, enquanto as histórias de quadrinhos de super-heróis alcançavam um número de apenas 20 mil exemplares, e os da Disney com menos de 30 mil.

Outro microgrupo presente no Japão, que surgiu dessa paixão pelo animê e mangá e se espalhou de forma intensa no mundo todo são os otakus. Os otakus tem uma ligação tão forte com os elementos que compõem o animê media, que Azuma (2001) classifica como uma subcultura, ou a cultura otaku. Grande parte dos consumidores brasileiros que adquirem os produtos de mídia nipônica faz parte desta subcultura. Eles são marcados pelo colecionismo (NAGADO, 2005) e consumismo (AZUMA, 2001); alguns incorporam personagens e imitam suas vestimentas e até características psicológicas (cosplayers). Ao contrário do perfil otaku japonês, que se mostra tímido e antissocial, o otaku brasileiro distancia-se dessa imagem, e mantém sua própria essência (CARLOS, 2011). A respeito disso, Nagado (2005) afirma:

\begin{abstract}
O público brasileiro é formado por muitas garotas e casais de namorados otakus, o que seria uma contradição no Japão. Muito mais soltos, entusiastas e barulhentos do que suas contrapartes orientais, os fãs brasileiros se acotovelam por um autógrafo de seu dublador preferido, pulam ouvindo animê songs como se estivessem em um show de rock e promovem uma confraternização bem brasileira, que certamente, estão distantes do fanatismo solitário e isolado presente em muitos otakus japoneses (NAGADO, 2005).
\end{abstract}

O principal meio de se socializar e consumir a cultura otaku, no Brasil há eventos que reúnem diversos conteúdos a respeito deste universo, dentre eles podemos citar o Animê Friends que ocorre anualmente em São Paulo, organizado pela empresa privada de eventos voltada para a cultura pop oriental, Yamato Corporation, e também o evento Cool Japan que ocorreu em 2009 no Rio de Janeiro e foi organizado em conjunto pela Academia Brasileira de Letras, Instituto Japão Pop BR e Consulado do Japão (MADE IN JAPAN, 2009). Em São Paulo, o bairro da Liberdade, uma área de relevância, pois remete à imigração dos japoneses ao Brasil, é o paraíso para os otakus brasileiros, onde é possível consumir e vivenciar a experiência da cultura pop japonesa. Paralelamente, com a ascensão da Internet, o consumo desses produtos midiáticos japoneses aumentou devido a atividades colaborativas entre fãs, que disponibilizam e traduzes episódios das animações online.

Pode-se classificar o consumidor otaku brasileiro como um consumidor-artesão, que se baseia na personalização e/ou humanização da mercadoria; investindo sua 
subjetividade e criatividade no objeto; e é oposto do consumidor passivo. Esse tipo de consumo proporciona experiências agradáveis aos consumidores, e o torna envolvido na concepção e produção do objeto a ser consumido (CAMPBELL, 2004). A grosso modo, é uma experiência de consumo através da humanização de objetos da mídia de massa, propiciando a eles um toque pessoal e subjetivo. Eles não consomem somente o produto em si, mas toda uma cultura.

\section{Conclusão}

Apesar do número considerável de consumidores brasileiros de produtos midiáticos japoneses, a oferta ainda é pequena se comparada à demanda. $\mathrm{O}$ consumo de produtos culturais japoneses pelos brasileiros inicia-se pelos animês e mangás, e isto leva posteriormente para o consumo da música e da moda. O conteúdo de animê media é disponibilizado, em sua maior parte, pela internet por meio de serviços colaborativos entre fãs. No ano de 2008 , o Brasil correspondia a menos de $10 \%$ do total mundial do mercado consumidor legal de animês e produtos relacionados (SHIMANOUSHI, 2009). Analisando o potencial de aceitação e consumo desses produtos no Brasil, acreditamos que ainda há possibilidade de uma larga ampliação desse mercado para essa indústria.

O governo do primeiro ministro, Shinzo Abe, até 2014, pretendia impulsionar as indústrias criativas do Japão no exterior, com um orçamento de US\$ 883 milhões. Uma das medidas tomadas pelo governo para penetrar a cultura japonesa pelo mundo tem sido o investimento em filmes e em canais de TV com programação japonesa voltada para o público ocidental, como o canal Waku Waku TV; por enquanto o Brasil ainda não foi cotado para transmiti-lo. A iniciativa teve origem com a observação do sucesso da Coréia do Sul em exportar suas novelas e músicas para o restante do mundo (THE ECONOMIST, 2014).

Uma das principais queixas de fãs de Animê Media no Brasil é que animês e mangás sofrem distorções em suas traduções e edições/cortes de cenas, pois fora originariamente produzido para o público jovem (MALONE, 2006). O problema consiste no fato de que as emissoras brasileiras direcionam para o público infantil os animês, de forma que estão sujeitas a obedecer as leis referentes à faixa etária. Os otakus brasileiros, em sua maioria, possuem um perfil jovem de 12 a 25 anos, pertencentes às classes A e B.

$\mathrm{Na}$ linguagem de mercado, podemos dizer que a estratégia do Cool Japan, visa a um reposicionamento da marca Japão. Segundo Telles (2004, p.73), reposicionamento é "[...] a ação de redefinir os elementos da identidade de marca a serem ativamente comunicados para um dado público-alvo". Sendo assim, o Japão pode ter conseguido obter uma imagem positiva do país em terras brasileiras através de seus esforços de soft power com a estratégia de Cool Japan. Essa imagem positiva pode estar sendo refletida não só no Brasil, mas também em outros países. Aso, o ex-primeiro ministro japonês, mencionou a atitude positiva que uma grande parte dos países tem com o Japão, obtendo 
boas classificações sobre o seu brand strength. Assim como uma empresa, um país também depende de uma gestão que trabalhe uma imagem positiva para prosperar e realizar exportações de seus produtos (ANHOLT, 2010).

\section{Referências Bibliográficas}

AAKER, D.A. Building Strong Brands, New York: The Free Press. 1996.

ALBUQUERQUE, Carlos. No Japão, o CD resiste: venda de música em compact disc ainda responde por $85 \%$ do mercado. O Globo, São Paulo. 19 jan. 2015. Disponível em:<http:// oglobo.globo.com/cultura/no-japao-cd-resiste-venda-de-musica-em-compact-disc-aindaresponde-por-85-do-mercado-15088198>. Acesso em: 30 mai. 2015.

ALLISON, Anne. The cool brand, affective activism and Japanese youth. Theory, Culture \& Society. Los Angeles: Sage, 2009. p. 89-111.

ALMEIDA, Paulo Roberto de. As últimas décadas do século XX: fim do socialismo e retomada da globalização. In: SARAIVA, José Flávio Sombra (Org.). História das Relações Internacionais Contemporâneas: da sociedade internacional do Século XIX à era da globalização. São Paulo: Saraiva, 2008, p. 253-316.

ANHOLT, Simon. Nation Brand Hexagon. Citado en Kemming, Jan Dirk. Nation Brand Management in Political Contexts: Public Diplomacy for Turkey's EU Accession. Giessen, 2009. p.21.

. Definitions of Place Branding: Working Towards a Resolution, Place Branding and Public Diplomacy, 6 Vol., 1-10, 2010.

ASO, Taro. Diplomacia do Japão: Garantindo a Segurança e a Prosperidade. Japan Institute of International Affairs, 30 jun. 2009. Discurso Proferido por Sua Ex ${ }^{a}$ Sr. Taro Aso, Primeiro-Ministro do Japão. Disponível em: <http://www.mz.emb-japan.go.jp/ Garantindo\%20a\%20Seguranca.pdf.> Acesso em 22 de abril de 2015.

AZUMA, Hiroki. Otaku: Japan's Database Animals. Minnesota Press, 2001.

BRASIL. Brasil e Japão assinam 9 acordos de cooperação internacional. Portal Brasil. Brasília, 01 ago. 2014. Disponível em: <http://www.brasil.gov.br/governo/2014/08/brasil-e-japaoassinam-9-acordos-de-cooperacao-internacional>. Acesso em 23 de abril de 2015.

CAMPBELL, Colin. O consumidor artesão: cultura, artesania e consumo em uma sociedade pós-moderna. Antropolítica, Niterói, n. 17, p.45-67, 2. sem. 2004.

CARLOS, Giovana. O(s) fã(s) da cultura pop japonesa e a prática de Scanlation no Brasil. Dissertação de Mestrado. PPG Comunicação e Linguagens, UTP, Curitiba, 2011.

DINNIE, K. Nation Branding: Concepts, Issues, Practice, Oxford, Butterworth Heinemann, 2008.

FAN, Y., Branding the Nation: What is Being Branded?. Journal of Vacation Marketing, 12, 2006. 
, Branding the Nation: Towards a Better Understanding, Place Branding and Public Diplomacy, 6 Vol., 98-100, 2010.

FLATH, David. The Japanese economy. Oxford, New York. Oxford University Press, 2005.

GRAIEB, C. O Japão é pop. VEJA. n. 1835, 7 jan. 2004. Disponível em: < http://www.abrademi. com/?p=505>. Acesso em: 25 mai. 2015.

GRAVETT, Paul. Mangá: como O Japão Reinventou Os Quadrinhos. São Paulo: Conrad Editora do Brasil, 2006.

GOTO, Marcel R. A história do mangá no brasil. Revista Herói, São Paulo, 2003.

Governo do Estado de São Paulo. 1908-2008:100 anos Imigração Japão Brasil. 2008. Disponível em: <http://www.saopaulo.sp.gov.br/imigracaojaponesa/historia.php $>$. Acesso em: 20 abr. 2015.

GUSMAN, Sidney. Mangá : hoje, o único formador de leitores do mercado brasileiro de quadrinhos. In: LUYTEN, Sonia B. (Org.); Cultura pop japonesa: mangá e animê. São Paulo: Hedra, 2005. p. 79-84.

HIGHLIGHTING JAPAN. Japan's pop culture broadens its international reach. v.3, n.5, set. 2009. Disponível em: <http://www.gov-online.go.jp/pdf/hlj_ar/ vol_0026e/10-13.pdf>. Acesso em: 8 jan. 2015.

HONG, Euny. Soap, sparkle and pop. The Economist Newspaper, Londres, 9 ago. 2014. Disponível em: < http://www.economist.com/node/21611039>. Acesso em: 7 fev. 2015.

JAPAN, METI (Ministry of Economy, Trade and Industry). Cool Japan Initiative. 2014. Disponível em: <http://www.meti.go.jp/policy/mono_info_service/mono/creative/ file/1406CoolJapanInitiative.pdf > . Acessado em: 7 jun. 2015.

KENMOCHI, Hideki. VOCALOID and Hatsune Miku phenomenon in Japan. Tokyo, 2010.

LUYTEN, Sônia M. Bibe. Mangá produzido no Brasil: pioneirismo, experimentação e produção, In: XXVI CONGRESSO BRASILEIRO DE CIÊNCIAS DACOMUNICAÇÃO. Belo Horizonte: INTERCOM, 2003.

MACIAS, Patrick; EVERS, Izumi. Japanese Schoolgirl. Inferno. São Francisco: Chronicle Books, 2007.

MALONE, Verena. Desenho-animado One Piece estréia em versão editada no Brasil. Made in Japan. São Paulo, 03 mai. 2006. Disponível em: $<$ http://madeinjapan.uol.com. br/2006/05/03/desenho-animado-one-piece-estreia-em-versao-editada-no-brasil/>. Acesso em: 7 abr. 2015.

MCGRAY, Douglas. Japan's gross national cool. Foreign Policy Magazine, 2001. 10p.

MDIC (Ministério do Desenvolvimento, Indústria e Comércio do Brasil). Balança comercial brasileira: dados consolidados, 2014.

MONJI, K. Pop culture diplomacy. Public Diplomacy Magazine. v.3, p.104-108, winter, 2010. NAGADO, Alexandre. Almanaque da Cultura Pop Japonesa. São Paulo: Via Lettera, 2007. 
. O mangá no contexto da cultura pop japonesa e universal. In: LUYTEN, Sonia B.

(Org.); Cultura pop japonesa: mangá e animê. São Paulo: Hedra, 2005.

NYE, Joseph S. Soft power. Foreign policy, 1990, p. 153-171.

OKA, Arnaldo Massato. Mangás traduzidos no Brasil. In: LUYTEN, Sonia B. (Org.); Cultura pop japonesa: mangá e animê. São Paulo: Hedra, 2005. p. 85-94.

ONOUCHI, M. General introduction to manga, animê and game. In: JAPÃO. EMBASSY OF JAPAN, LONDON. (Ed.). Creative Japan, 2007. p. 4-7.

PEREIRA, Paulo Gustavo. Almanaque dos seriados. São Paulo: Ediouro, 2008.

SATO, C. A. JAPOP: o poder da cultura pop japonesa. NSP-Hakkosha: São Paulo, 2007.

SHIMANOUSHI, Ken. A crise econômica mundial e a relação bilateral Brasil-Japão. 2009. Palestra realizada no dia 13/05 na sede da Fiesp, São Paulo. Disponível em: <http:// pt.camaradojapao.org.br/camara-em-acao/opiniao/?materia=7251>. Acesso em: 30 mai. 2015.

SQUARING the Cool. The Economist, Tokyo, 16 Jun. 2014. Disponível em: <http://www. economist.com/blogs/banyan/2014/06/japans-soft-power>. Acesso em: 18 abr. 2015.

SNOW, N.; TAYLOR, P. M. (Eds). Routledge handbook of public diplomacy. New York: Routledge, 2008.

TELLES, R. Posicionamento e reposicionamento de marca: uma perspectiva estratégica e operacional dos desafios e riscos, 2004. Tese (Doutorado em Administração) - PPGAFEA/USP.

UEHARA, Alexandre Ratsuo. A política externa do Japão no final do século XX: o que faltou?. São Paulo: Annablume, 2003

XXI. Fundação Japão em São Paulo, 2012.

VILIEGAS, Renato. Se não fosse por eles... Henshin. São Paulo, n. 21: 18-22, 2001.

VOGEL, Ezra F. Japan as number one: lessons for America. Cambridge: Harvard University Press, 1979.

YOSHIMOTO, Mitsushiro. The status of creative industries in Japan and policy recommendations for their promotion. Social development research group, 2003 